DrAft VERSION OCTOBER 17, 2019

Preprint typeset using $\mathrm{LAT}_{\mathrm{E}} \mathrm{X}$ style emulateapj v. 08/22/09

\title{
INSIGHT-HXMT OBSERVATIONS OF 4U 1636-536: CORONA COOLING REVEALED WITH SINGLE SHORT
} TYPE-I X-RAY BURST

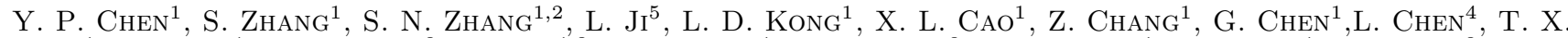

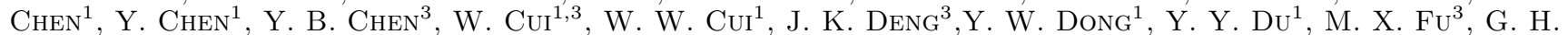

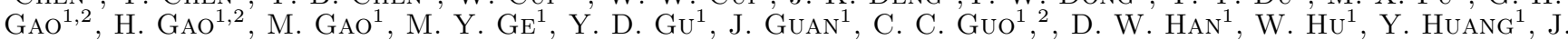

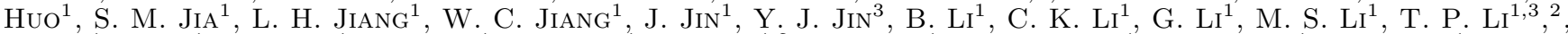

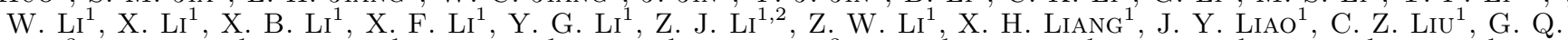

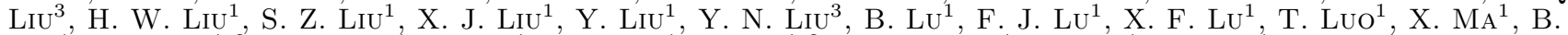

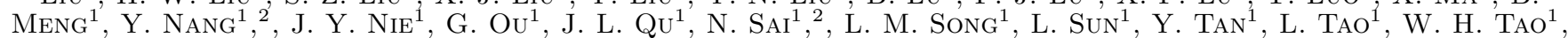 \\ Y. L. TUO ${ }^{1,2}$, G. F. WANG ${ }^{1}$, H. Y. WANG ${ }^{1}$, J. WANG ${ }^{1}$, W. S. WANG ${ }^{1}$, Y. S. WANG ${ }^{1}$, X. Y. WEN ${ }^{1}$, B. B. WU $^{1}$, M. WU $^{1}$,

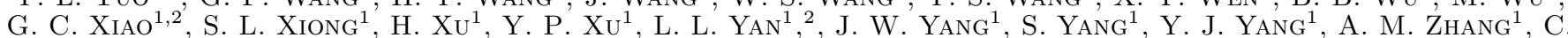

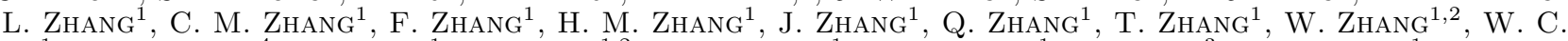

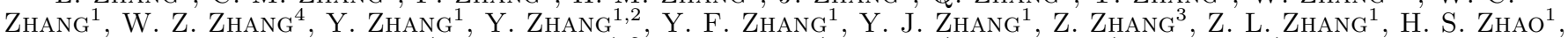 \\ J. L. ZHAO ${ }^{1}$, X. F. ZHAO ${ }^{1}{ }^{2}$, S. J. ZHenG ${ }^{1}$, Y. ZHU ${ }^{1}$, Y. X. ZHU ${ }^{1}$, C. L. ZOU ${ }^{1}$ \\ ${ }^{1}$ Key Laboratory for Particle Astrophysics, Institute of High Energy Physics, Chinese Academy of Sciences, 19B Yuquan Road, Beijing \\ 100049, China \\ ${ }^{2}$ University of Chinese Academy of Sciences, Chinese Academy of Sciences, Beijing 100049, China \\ 3 Department of Physics, Tsinghua University, Beijing 100084, China \\ ${ }^{4}$ Department of Astronomy, Beijing Normal University, Beijing 100088, China and \\ ${ }^{5}$ Institut für Astronomie und Astrophysik, Kepler Center for Astro and Particle Physics, Eberhard Karls Universität, Sand 1, D-72076 \\ Tübingen, Germany \\ Draft version October 17, 2019
}

\begin{abstract}
Corona cooling was detected previously from stacking a series of short type-I bursts occurred during the low/had state of atoll outburst. Type-I bursts are hence regarded as sharp probe to our better understanding on the basic property of the corona. The launch of the first Chinese X-ray satellite Insight-HXMT has large detection area at hard X-rays which provide almost unique chance to move further in this research field. We report the first detection of the corona cooling by Insight-HXMT from single short type-I burst showing up during flare of $4 \mathrm{U}$ 1636-536. This type-I X-ray burst has a duration of $\sim 13$ seconds and hard X-ray shortage is detected with significance $6.2 \sigma$ in 40-70 keV. A cross-correlation analysis between the lightcurves of soft and hard X-ray band, shows that the corona shortage lag the burst emission by $1.6 \pm 1.2 \mathrm{~s}$. These results are consistent with those derived previously from stacking a large amount of bursts detected by RXTE/PCA within a series of flares of $4 \mathrm{U}$ 1636-536. Moreover, the broad bandwidth of Insight-HXMT allows as well for the first time to infer the burst influence upon the continuum spectrum via performing the spectral fitting of the burst, which ends up with the finding that hard X-ray shortage appears at around $40 \mathrm{keV}$ in the continuum spectrum. These results suggest that the evolution of the corona along with the outburst/flare of NS XRB may be traced via looking into a series of embedded type-I bursts by using Insight-HXMT.

Subject headings: stars: coronae — stars: neutron - X-rays: individual(4U 1636-536) - X-rays: binaries - X-rays: bursts
\end{abstract}

\section{INTRODUCTION}

Type-I X-ray burst, also named thermonuclear burst (hereafter burst), is caused by unstable burning of the accreted hydrogen/helium on the surface of a neutron star (NS) enclosed in an X-ray binary (XRB), and manifest themselves as a sudden increase (typically by a factor of 10 or greater) in the X-ray luminosity followed by an exponential decay (for reviews, see Lewin et al. 1993; Cumming 2004; Strohmayer \& Bildsten 2006; Gallowav et al. 2008). The most luminous bursts are the photospheric radius expansion (PRE) events, for which the peak flux is comparable to the Eddington luminosity at the surface of the NS.

More and more observational examples and theory models on the burst influence on the persistent/accretion emission have rapidly accumulated over recent years (Degenaar et al. 2018). Basically, there are three obser-

Electronic address: chenyp@ihep.ac.cn, szhang@ihep.ac.cn vational evidence and corresponding physical processes initiation between the two kinds emission: accretion rate change because of Poynting-Robertson drag induced by dynamic/light-pressure of burst; disk or/and corona change both in structural or/and intrinsic characteries owing to the burst cooling/heating; enhancement emission result from refection of the accretion disk. Among the observation evidences, most of them are from intermediate long burst/super-burst or stacking lots of normal burst (Degenaar et al. 2018).

For the interaction between bursts and corona, the shortage in the hard X-ray of the continuum emission is reported on several XRB, i.e., IGR J174732721 (Chen et al. 2011; Chen et al. 2012), Aql X-1 (Chen et al. 2013; Maccarone \& Coppi 2003), 4U 1636536 (Ji et al. 2013), GS 1826-238 (Ji et al. 2014a), KS 1731-260 (Ji et al. 2014b), 4U 1705-44 (Ji et al. 2014b) and 4U 1728-34 (Kajava et al. 2017), based on RXTE/PCA and INTEGRAL observation. It is very 
hard to detect the deficit up to $5 \sigma$ in single short burst because of the relatively small detection area of the previously missions at hard X-ray, and hence these firm detection significance are aggrandized by stacked tens to hundreds bursts. For example, 4U 1636-536, the deficit detection is based on $\mathbf{3 6}$ bursts results.

In the timing analysis zone, effects of X-ray bursts on $\mathrm{kHz}$ QPOs are also revealed in several bursters such as Aql X-1 (Yu et al. 1999) and 4U 1636-536 (Peille et al. 2014), often manifests as QPO frequency and persistent flux changes between before and after the burst. Such detections are interpreted that the burst blew away or pulled up the inner disk based on the different viscous time-scale for QPO recovery.

4U 1636-536, a low-Mass X-ray Binary (LMXB), discovered with the 8th Orbiting Solar Observatory (OSO8; Swank et al. 1976), it is a well-studied LMXB which holds an 18th magnitude blue star, V801 Ara in an orbit of $3.8 \mathrm{hr}$ (van Paradijs et al. 1990). It is one of the few persistent X-ray sources in our Galaxy that undergoes regularly transitions between the hard state and the soft state, in a repeating period of roughly 70 days, and many bursts accompanied. The properties of the burst oscillations of $579.3 \mathrm{~Hz}$ and super-bursts with hours duration have been detected and analyzed (see Galloway et al. 2008 for a review). From its colour-color diagram (CCD), 4U 1636-536 traces a U-shape or Cshape as a typical atoll source (detailed CCD was shown in Zhang et al. 2011). Its distance was estimated as $\sim 6 \mathrm{kpc}$ (Galloway et al. 2006) by using the PRE burst.

In this work we study the burst-corona interaction in the LMXB 4U 1636-536 using the first year of data collected with Insight-HXMT Zhang et al. 2014. In Sect. 2 we present Insight/HXMT observations and data analysis procedure by detail. In Sect. 3, the shortage in hard $\mathrm{X}$-ray band are given based on the broad-band spectrometry results. In Sect. 4, we present our interpretation on the detection and the comparison with previous detection on the hard X-ray shortage during burst.

\section{OBSERVATIONS AND DATA ANALYSIS}

On June 15th of 2017, the Hard X-ray Modulation Telescope (HXMT, also dubbed as InsightHXMT Zhang et al. 2014)was launched in Jiuquan Satellite Launch Center. It excels in broad energy band (1-250 keV) detection ability and large effective area in hard X-rays energy band. It consists of three slat-collimated instruments: the High Energy X-ray Telescope (HE), the Medium Energy X-ray Telescope (ME), and the Low Energy X-ray Telescope (LE), with collecting-area/energy-range in $\sim 5000 \mathrm{~cm}^{2}$ in 20-250 $\mathrm{keV}, \sim 900 \mathrm{~cm}^{2}$ in $5-30 \mathrm{keV}$ and $\sim 400 \mathrm{~cm}^{2}$ in $1-10 \mathrm{keV}$ respectively.

In this work, we analyze the brightest of three bursts, represent the time-resolved spectroscopy and give our interpretation on the uniqueness observational hehavior. By virtue of quick read-out time of Insight-HXMT detectors, there is little pile-up event at the PRE burst peak. HEASOFT version 6.22.1 and Insight-HXMT Data Analysis software (HXMTDAS) v2.01 were used to process and analyze the data. Only the small field of view (FoV) of $\mathrm{LE}$ and ME were used, because large FoV were easily contaminated by near-by source and the bright earth. The good time interval were filterd with the following criteria: (1) pointing offset angles $<0.05$ degree; (2) elevation angles $>6$ degree; $(3)$ the value of the geomagnetic cutoff rigidity $>6$.

Among its first years observations, the total InsightHXMT observation of $4 \mathrm{U} 1636-536$ is $\sim 370 \mathrm{ks}$, covering the time span between February 11th and July 1st of 2018. Eight type-I X-ray burst:4are detected in 4U 1636536. Among them, the first three burst have low flux level of persistent both in soft at $\sim 40 \mathrm{mCrab}$ and hard $\mathrm{X}$-ray at $\sim 15 \mathrm{mCrab}$. The other five bursts have high hard X-ray flux and relatively low flux in soft X-ray, indicates it locates island state (similar with low/hard state of black hole XRB). Most of them are lack of LE results because of optical pollution from the bright earth. Form these eight bursts, we choose a burst which satisfies the good-time-interval selection criteria of LE, $\mathrm{ME}$ and $\mathrm{HE}$ simultaneously, and locates at the island state with a high hard X-ray flux $\sim 75$ mCrab of its inhabited persistent emission. (Fig 1) The obsid number is P011465402801-20180701-01-01, with peak flux happened at MJD 58300.717896.

The lightcurve profile of the burst is derived in time bins of $1 \mathrm{~s}$ in the full passband of LE\&ME and 40-70 $\mathrm{keV}$ of $\mathrm{HE}$, with pre-burst emission subtracted, is shown in Fig 2 the top, middle and bottom pad for LE, ME and HE respectively. From Fig. 2, the lightcurves are stable before and after the burst, indicating little variation of the persistent and background emission.

We adopted the standard analysis procedure of burst, i.e., take pre-burst emission (including instrumental background and persistent/accretion flux of the neutron star system) as background to investigate the burst spectra evolution. We divide the burst into intervals of 1 seconds after the burst onset, and extract the spectra of $\mathrm{LE}, \mathrm{ME}$ and $\mathrm{HE}$ respectively. For the burst, we use the time of the bolometric flux peak as a reference $(0$ second in Fig 3) to produce the lightcurve/spectra. A blackbody model (bbodyrad in Xspec) with fixed absorption $0.41 \times 10^{22} \mathrm{~cm}^{-2}$ as derived in Agrawal\& Hasanet (2016) is used to fit the burst spectra. To compromise the effective area calibration deviation, a constant is added to the model. At first attempt, for LE, the constant is fixed to 1, the others (ME and $\mathrm{HE}$ ) are alterable during spectra fitting. But the fitting result indicate that most of the constant of $\mathrm{HE}$ are not convergent, owing to the low-significance of the HE detection. Under this situation, the constant of $\mathrm{HE}$ was fixed at 1 for the combinedspectra fitting, which based on combined-spectra fitting of Crab observations by the same detector selection. The unabsorbed bolometric flux of the spectra is estimated by the XSPEC model cflux, and the observed blackbody radius is estimated under condition that the distance of $4 \mathrm{U} 1636-536$ is $6 \mathrm{kpc}$. The fitting results are shown in Fig 3 and Fig 4.

\section{RESULTS}

\subsection{Lightcurves of burst emission in soft and hard $X$-ray band}

As shown in Fig. 2, the HE flux is mostly negative during the burst and around zero elsewhere. This deficit is $12.4 \pm 2.0 \mathrm{cts} / \mathrm{s}$, and its significance is $6.2 \sigma$, which is estimated by the ration of the deficit area and the sum of the error-bars with duration of 32 seconds. The pre- 
burst emission of HE is $\sim 140$ counts/s in $40-70 \mathrm{keV}$, which include the background $\sim 125$ counts/s and persistent emission $\sim 15$ counts/s. From Fig. 2, the hard X-ray decrement reaches a maximum of $16.1 \pm 5.5 \mathrm{cts} / \mathrm{s}$ at the soft-X-ray burst peak. Considering the two values above, we conclude that almost all of the persistent emission in $40-70 \mathrm{keV}$ is crippled at the burst peak-time.

A cross-correlation analysis is attempted between the LE lightcurves in 1.1-12 keV and re-extracted HE lighcurve in $40-70 \mathrm{keV}$ with a bin size of $1 \mathrm{~s}$, as shown in Fig,2, The minimum of the cross-correlation value appears at $1.6 \pm 1.2 \mathrm{~s}$, indicated that hard X-ray deficit delays the burst emission. The value is derived from Gaussian-fitting of Fig,2,

\subsection{Broad-band spectra of burst emission}

The time-ordered burst spectra fitting results are as shown in Fig 3. the peak bolometric flux is $2.6 \pm 0.4 \times$ $10^{-8} \mathrm{erg} \mathrm{cm}^{-2} \mathrm{~s}^{-1}$, which is roughly half of the Eddington luminosity $6.0 \pm 0.6 \times 10^{-8} \mathrm{erg} \mathrm{cm}^{-2} \mathrm{~s}^{-1}$ obtained from the PRE bursts of obsid P011465400301-2018021301-01. The reduced $\chi^{2}$ of the jointed spectra fitting are roughly at 1 , with most of the degree of freedom more than 50. The duration of the bursts is also estimated from the ration of the bolometric flux to the peak flux, $\sim 13.1$ s.

In the process of fitting 1-s exposure jointed spectra, by taking pre-burst emission as background, while the residual of LE and ME are roughly around 0, a marginally deficit appears for HE. For easy reading, all the timeordered spectral fitting results in Fig 3 are merged into one graph which is shown in Fig. 4, a clear deficit is visible around 40-50 keV. To investigate further the deficit, a jointed spectra with 32 seconds exposure time is extracted, and fitted with two blackbody model (with absorption fixed). The consideration of one more blackbody spectral component is to account for the temperature evolution in case of handing the entire burst. The embedded HE spectra of Fig. 4 shows that the negative values appear at $40-70 \mathrm{keV}$, the significance of this deficit in $40-70 \mathrm{keV}$ from the spectra is $6.8 \sigma$. This value is estimated based on the different counts of the bursts and pre-burst emission from the spectra, i.e., 4012 counts for the burst and 4465 counts for pre-burst emission at the same energy band. From the above analysis, the significance of the deficit derived from the spectral and lightcurves are consistent each other.

\section{DISCUSSION}

Observations of Insight/HXMT, provide us so far the best chance to study the effects of type-I X-ray burst on continuum emission of NS XRB, thanks to the large detection area and wide bandwidth of the Insight-HXMT. Here with the Insight-HXMT we find for the first time the hard X-ray deficit/shortage via single short typeI burst showing up during flare of $4 \mathrm{U}$ 1636-536. The hard X-ray shortage is detected with significance 6.2 in 40-70 keV, and a cross-correlation analysis between the lightcurves of soft and hard X-ray band, shows that the corona shortage lag the burst emission by $1.6 \pm 1.2 \mathrm{~s}$. These results are consistent with those derived previously from stacking a large amount of bursts detected by XTE/PCA within a series of flares of $4 \mathrm{U}$ 1636-536. Moreover, the broad bandwidth of Insight-HXMT allows as well for the first time to infer the burst influence upon the continuum spectrum via performing the spectral fitting of the burst, which ends up with the finding that hard X-ray shortage appears at around $40 \mathrm{keV}$ in the continuum spectrum. These results suggest that the evolution of the corona along with the outburst/flare of NS XRB may be traced via looking into a series of embedded type-I bursts by using Insight-HXMT.

Our previous work has revealed the deficit of the hard X-rays in six sources using RXTE/PCA data, by constructing a sample with tens bursts for each source (e.g., Chen et al. 2012; Ji et al. 2013, and reference therein). Since all these reports are based on the RXTE/PCA observations, a suspicion may arise for the dead time concerning which may have influence upon the significance of observing a hard X-ray shortage accompanied with the type-I burst. Although later on INTEGRAL observations also confirm such kind of deficit in 4U 1728-34 (Kajava et al. 2017), through stacking a sample of 123 bursts in low/hard state, but they reported detection significances of $3.4 \sigma$ in the $40-50 \mathrm{keV}$ band and $1.8 \sigma$ in the $50-80 \mathrm{keV}$ band. In this work, benefitting the broad energy coverage of three different detectors, this phenomenon is also detected for the first time with a single in single burst of $4 \mathrm{U}$ 1636-536.

As were discussed in previous papers, the deficit in hard X-rays likely indicates a cooling of the corona by the burst, which provides an intense shower of the soft $\mathrm{X}$-rays to cool the hot corona via Compntonization. The time lag between the burst at soft X-rays and the deficit at hard X-rays for the continuum emission is considered as the timescale of the corona reheating/reformation. So far all the time-lags of the deficits detected in the above sources are within several seconds, indicating a similar mechanism for corona production during outburst/flare of NS XRBs.

As shown in Table 4 and Fig. 1, apart form the burst with a hard X-ray shortage clearly seen, there are other three bursts detected during the soft state of the $4 \mathrm{U} 1636$ 536. Two of them are the short type-I bursts, and one is the PRE burst. No hard X-ray shortages are detected for the continuum emission during these bursts, due to that the continuum hard X-ray emissions are too weak. As shown in the Swift/BAT observations, the source stayed at roughly $\sim 15 \mathrm{mCrab}$ level at above $15 \mathrm{keV}$ at time around these three bursts. The spectral analysis of the these bursts, show no obvious significant residual detected in LE\&ME spectra fitting results.

Although a hint of excess appears at below $\sim 2 \mathrm{keV}$ for the burst showing up in the hard state of the flare, it becomes invisible by setting the absorption parameter free during spectra fitting. Usually, the persistent emission change are detected in RXTE/PCA and NICER, and the degree of enhancement is proportional to the burst flux. The faint of the burst peak flux probably prevents us to detect the effects of the bursts on the disk emission. Clear residuals below $\sim 2 \mathrm{keV}$ are detected at the PRE burst of obsid P011465400301-20180213-01-01, which is detected with faint persistent emission, also indicates the state-depended of the persistent emission enhancement induced by the burst. The PRE burst results will be reported by the upcoming paper.

The physical origin of QPOs at $\mathrm{kHz}$ is thought to be the dynamical timescale of the inner part of the accre- 
TABLE 1

The Bursts obsid And PEAK time of $4 \mathrm{U}$ 1636-536 DEteCted By Insight/HXMT.

\begin{tabular}{ccc}
\hline No & obsid & Time (MJD) \\
\hline $1 *$ & P011465400301-20180213-01-01 & 58162.871091 \\
2 & P011465400401-20180215-01-01 & 58164.733723 \\
3 & P011465400501-20180217-01-01 & 58166.117179 \\
4 & P011465402301-20180626-01-01 & 58295.087021 \\
5 & P011465402701-20180630-01-01 & 58299.489308 \\
6 & P011465402801-20180701-01-01 & 58300.717896 \\
7 & P011465403101-20180704-01-01 & 58303.959479 \\
8 & P011465403201-20180705-01-01 & 58304.808113 \\
\hline
\end{tabular}

* Burst shows photosphere radius expansion.

tion disk and hence provide another way to diagnostic the burst influence upon the accretion disk/corona. For $4 \mathrm{U}$ 1636-536, QPO frequency changes during burst is detected(Peille et al. 2014). One interpretation is that the inner part of the disk is puffed up by the burst, which suppresses the QPO generation (Ballantyne \& Everett 2005). If the disk curls up to higher latitude, the deficit will become unremarkable because of cloaking the burst photons by the taller disk, since the disk is optically thick. The other interpretation to the QPO suppression is the backward/regression of the inner disk, i.e., $\mathrm{X}$-ray burst blow away the disk to behave bigger inner disk radius. However, the disk emission is detected to increase during burst by RXTE (in't Zand et al. 2013; Worpel et al. 2013; Ballantyne \& Strohmaver, 2004; Keek et al. 2014) and NICER (Keek et al. 2018), which indicates a smaller inner disk radius induced by burst. Unfortunately no QPO is detected from 4U 1636536 by the Insight-HXMT, probably due to its relatively small detection area at soft X-rays. An increase of disk temperature maybe reconcile this contradiction, in other word, the burst heats the disk.

The deficit energy covers a range of roughly $40-70 \mathrm{keV}$, extends to $\sim 70 \mathrm{keV}$, within which $\mathrm{HE}$ has the largest effective area. Considering the effective area of HE decreases in higher energy and low counts rate of the preburst emission for the continuum emission at hard Xrays, the deficit maybe both extends to lower/higher energies. More observations on the low/hard state of $4 \mathrm{U}$ 1636-536 and target of opportunity (ToO) observations on brighter burster, such as Aql X-1, may provide us better chance to investigate thoroughly both the time and spectral evolution of corona along with outburst/flare via investigating individual type-I burst with the unique capability of the Insight-HXMT. The upscattering photons of bursts by the continuum emission, should affect the shape/amplitude of blackbody spectra, joint observations on bursters by NICER or AstroSat and Insight/HXMT may give us opportunity to test the coronal cooling interpretation.

This work is supported by the National Key R\&D Program of China (2016YFA0400800) and the National Natural Science Foundation of China under grants 11473027, 11733009, U1838201 and U1838104.

\section{REFERENCES}

Agrawal, Vivek Kumar;\& Hasan, Mohammad, 2016, arXiv:1611.09004

Ballantyne, D. \& Everett, J., 2005, ApJ, 626, 364

Ballantyne, D. R., \& Strohmayer, T. E. 2004, ApJL, 602, L105

Chen, Y. P., Zhang, S., Torres, D. F. et al. 2011, A\&A, 510, 81

Chen, Y. P., Zhang, S., Zhang, S. N., et al. 2012, ApJL, 752, 34

Chen, Y. P., Zhang, S., Zhang, S. N., et al. 2013, ApJL, 777, 9

Cumming, A. 2004, Nucl. Phys. B Proc. Suppl., 132, 435

Czerny, M., Czerny, B., \& Grindlay, J. E. 1987, ApJ, 312, 122

Degenaar, N., Ballantyne, D. R., Belloni, T., et al. 2018, SSRv, 214,15

Galloway, D. K., Psaltis D., Muno M. P., Chakrabarty D., 2006, ApJ, 639, 1033

Galloway, D. K., Muno, M. P., Hartman, J. M., et al. 2008, ApJS, 179, 360

in't Zand, J. J. M., Galloway, D. K., Marshall, H. L., et al. 2013, A\&A,553, A83

Ji, L., Zhang, S., Chen, Y. P., et al., 2013, MNRAS, 432, 2773

Ji, L., Zhang, S., Chen, Y. P., et al., 2014, ApJ, 791, L39

Ji, L., Zhang, S., Chen, Y. P., et al., 2014, A\&A, 564, A20

Kajava, J. J. E., Sánchez-Fernández, C., Kuulkers, E., et al., A\&A, 599, 89

Keek, L., Ballantyne, D. R., Kuulkers, E., \& Strohmayer, T. E. 2014, ApJL, 797, L23
Keek, L., Arzoumanian, Z., Bult, P., et al. 2018, ApJL, 855,4 Lewin, W. H. G., van Paradijs, J., \& Taam, R. E. 1993, Space Sci. Rev., 62, 223

Maccarone, T. J. \& Coppi, P. S. 2003, A\&A, 399, 1151

Peille, P., Olive, J. F., Barret D., 2014, A\&A, 567, A80

Strohmayer, T., \& Bildsten, L. 2006, New views of

thermonuclear bursts (Compact stellar X-ray sources), 113, 156

Suleimanov, V.; Poutanen, J.; Werner, K. 2011, A\&A, 527, A139

Swank. J. H., Becker. R. H., Pravdo. S. H., Saba. J. R.,

Serlemitsos. P. J., 1976a, IAU Circ., 3000, 5

van Paradijs. J., van der Klis M., van Amerongen. S., et al., 1990, A\&A, 234, 181

Worpel, H., Galloway, D. K., \& Price, D. J. 2013, ApJ, 772, 94

Yu, W., Li, T.-P., Zhang. W., Zhang, S.-N., 1999, ApJL, 512, L35

Zhang. G. B., Méndez M., Altamirano. D., 2011, MNRAS, 413, $1913 Z$

Zhang. S., Lu. F. J., Zhang. S. N. et al.,in Space Telescopes and Instrumentation 2014: Ultraviolet to Gamma Ray, Proc. SPIE, Vol. 9144 (2014) p. 914421 


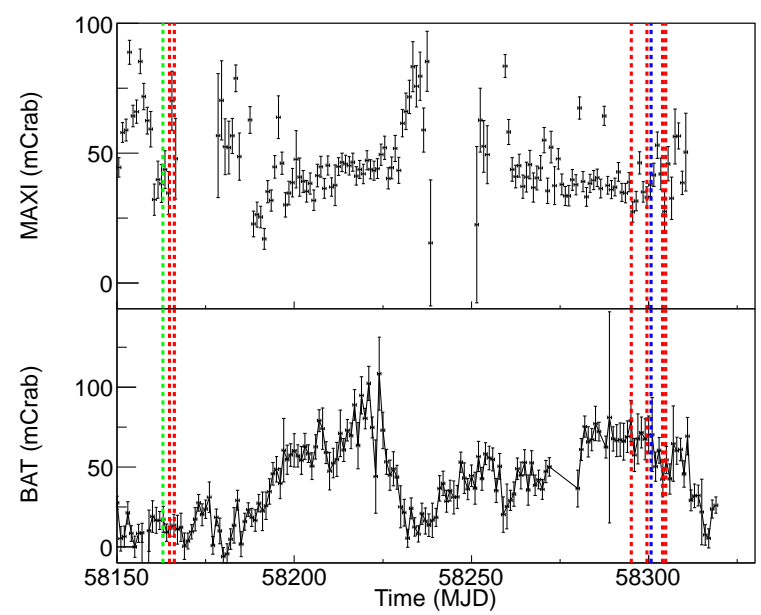

FIG. 1.- Daily lightcurves of 4U 1636-536 by MAXI and Swift/BAT during the outbursts in 2018, in 2-20 keV and 15-50 keV respectively. The burst is indicated by vertical lines, the PRE burst and the burst of this work are marked by green and blue respectively.
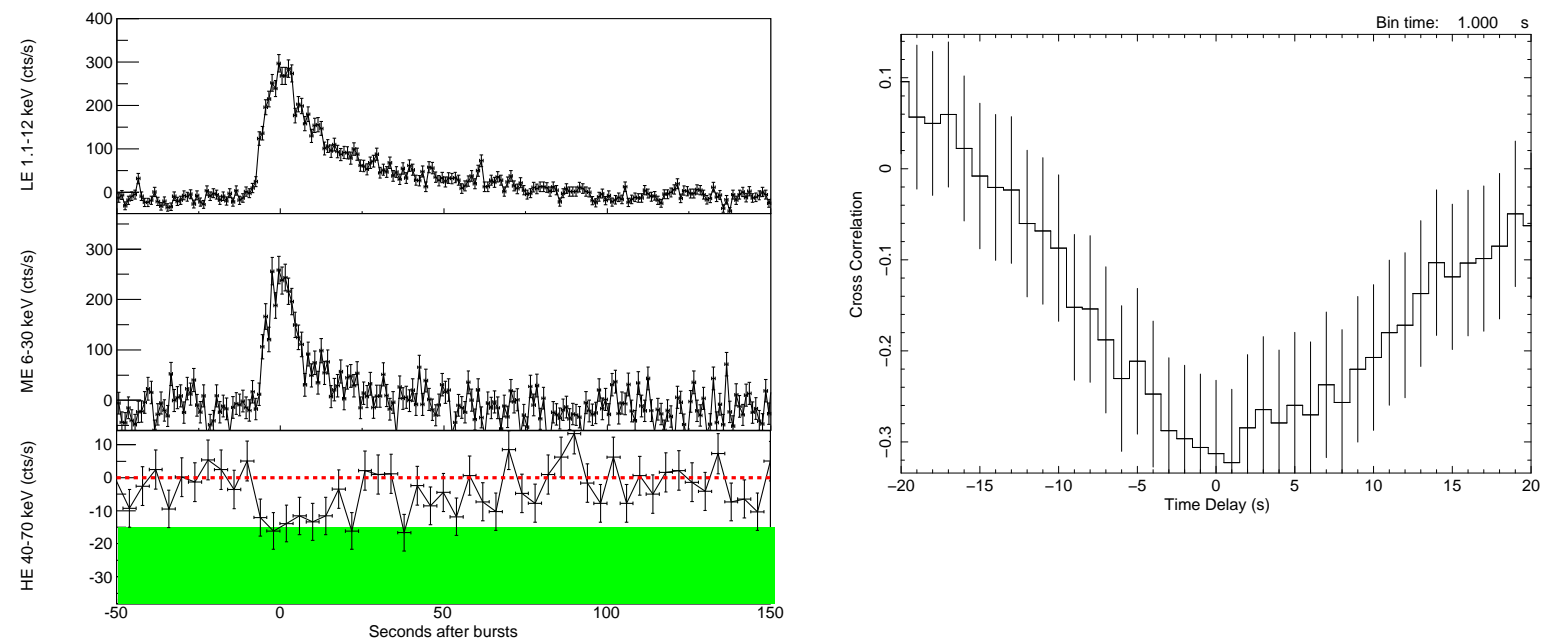

FIG. 2.- The left panel is the LE, ME and HE lightcurves of the burst in 1.1-12 keV, 5-30 keV and 40-70 keV respectively, the time-bin for LE\&ME is $1 \mathrm{~s}$ and $\mathrm{HE}$ is $4 \mathrm{~s}$, the green zone in bottom pad indicates the background level for HE detectors. The right panel shows the cross-correlation between the left panels's LE and HE re-extracted lightcurves with time bin $1 \mathrm{~s}$. 


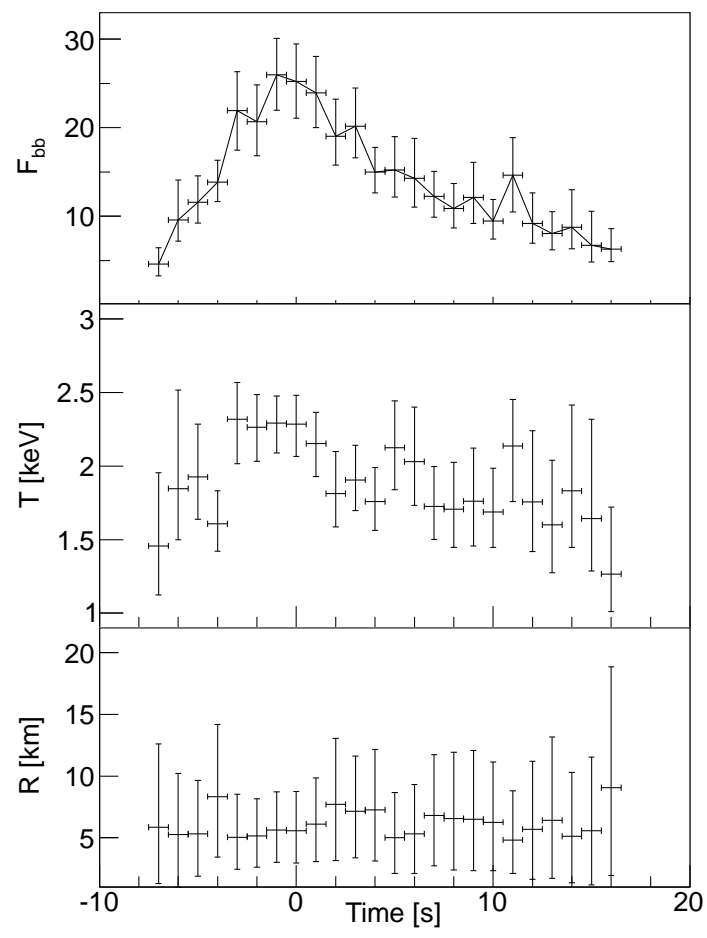

FIG. 3. - The time evolution of blackbody bolometric flux, the temperature T, the observed radius of NS surface at 6 kpc, with a time resolution of 1 second, the bolometric flux of blackbody $F_{b b}$ is in unit of $10^{-9} \mathrm{erg} / \mathrm{cm}^{2} / \mathrm{s}$.
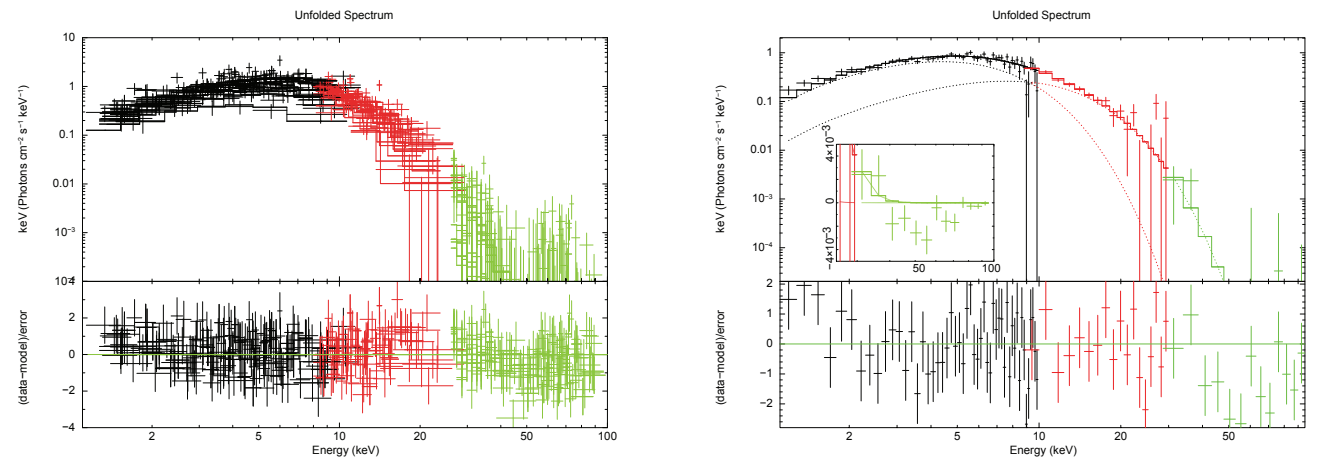

FIG. 4.- The left panel is merged the 1s exposure burst spectra fit results to one graph for easy reading. The right panel is $32 \mathrm{~s}$ exposure time spectra during the burst with model cons*wabs*(bbodryad+bbodyrad), the embedded panel shows HE spectra in detail since the negative value can not been shown in main panel with logarithmic coordinate axis. The enegy range of the spectra of LE (black), ME (red) and $\mathrm{HE}$ (green) is $1.1-12 \mathrm{keV}, 8-30 \mathrm{keV}$ and $25-100 \mathrm{keV}$ respectively. 